\title{
L'audit Social Comme Outil D'aide A La Gestion Des Ressources Humaines Des Organisations. Cas D'une Organisation Publique Et Une Autre Privée
}

\author{
Rachid Zammar \\ Professeur Habilité à la faculté des sciences de Rabat. \\ Université Mohammed V. Maroc \\ Noureddine Abdelbaki \\ Professeur Habilité à la faculté des sciences juridiques économiques et \\ sociales d'Agadir, Université Ibn Zohr. Maroc
}

Doi: 10.19044/esj.2017.v13n34p1 㞷L:http://dx.doi.org/10.19044/esj.2017.v13n34p1

\begin{abstract}
In a context marked by the increasing demands of all stakeholders for human resources policies and practices, expectations of social audit are increasing.

The objective of this research is to identify the scope of social audit and to show that it is a tool to assist human resources departments. It tries to address the question of how social auditing can help to anchor appropriate human resource management in an organization's practices.

To do this, a field survey was conducted among the staff of two organizations : one public (the urban district of Agadir) and the other private (Medium and Large ATLAS company) via semi-structured interviews.

The survey found that the introduction of social auditing in organizations is necessary since the latter has a beneficial effect on human resources management insofar as it ensures a positive social climate.
\end{abstract}

Keywords: Social audit, human resources, organization

\section{Résumé}

Dans un contexte marqué par les exigences croissantes de toutes les parties prenantes en matière de politiques et pratiques ressources humaines, les attentes à l'égard de l'audit social s'accroissent.

Ce travail de recherche s'assigne comme objectif de cerner le champ d'application de l'audit social et de montrer qu'il s'agit d'un outil d'aide aux services des ressources humaines. Il essaye de traiter de la question suivante : comment l'audit social peut-il contribuer à ancrer une gestion de ressources humaines appropriée dans les pratiques d'une organisation? 
Pour ce faire une enquête terrain a été menée auprès du personnel de deux organisations : une publique (la commune urbaine d'Agadir) et l'autre privé (société Moyen et Grand ATLAS) via des entretiens semi-directifs. L'enquête a permis de constater que l'introduction de l'audit social dans les organisations est nécessaire puisque ce dernier a un effet bénéfique sur la gestion des ressources humaines dans la mesure où il veille à garder un climat social positif.

Mots-clés: Audit social, ressources humaines, organisation

\section{Introduction}

Gérer une entreprise c'est savoir associer, coordonner et combiner les ressources (Penrose, 1958), non substituables (Barney, 1991), capables de créer de la valeur (Mahoney, 1995 ; Ireland et Hitt, 2002) et de l'avantage concurrentiel (Barney, 1991). Parmi ces ressources, on compte les ressources humaines dont l'usage pourrait induire en erreur si les outils utilisés ne sont pas adéquats.

Pour réduire ce risque, la plupart des entreprises recourent à l'audit social comme bonne conduite pour accompagner le renouvellement des politiques et pratiques des ressources humaines.

Ce travail de recherche s'assigne comme objectif de cerner le champ d'application de l'audit social et de montrer qu'il s'agit d'un outil d'aide aux directions des ressources humaines. Il essaye de traiter de la problématique suivante : comment l'audit social peut-il contribuer à ancrer une gestion de ressources humaines appropriée dans les pratiques d'une organisation?

Pour ce faire une enquête terrain a été menée auprès de deux organisations : une publique (la commune urbaine d'Agadir) et l'autre privé (société Moyen et Grand ATLAS).

\section{Cadre général de l'audit social}

\section{L'audit social : définition, missions et champs}

\section{Définition}

Selon Vatier (1980, p.25) l'audit social est un instrument de direction et de gestion et une démarche d'observation, qui, à l'instar de l'audit financier ou comptable, dans son domaine, tend à estimer la capacité d'une entreprise ou d'un organisation à maîtriser les problèmes humains ou sociaux que lui pose son environnement, et à gérer ceux qu'elle suscite elle-même par l'emploi du personnel nécessaire à son activité.

D'après Candau (1985, p.51), l'audit social est une démarche objective indépendante et inductive d'observation, d'analyse, d'évaluation, et de recommandation reposant sur une méthodologie et utilisant des techniques permettant, par rapport à des référentiels explicites, d'identifier, 
dans une première étape, les points forts, les problèmes induits par l'emploi du personnel, et les contraintes, sous formes de coûts et de risques. Ceci conduit à diagnostiquer les causes des problèmes décelés, à en évaluer l'importance et enfin à aboutir à la formulation de recommandations ou propositions d'action qui ne sont jamais mises en œuvre par l'auditeur.

Igalens et Peretti (2008, p.37), proposent la définition suivante : «L'audit social est un processus appliqué à la gestion, aux activités et aux relations des individus et des groupes dans les organisations, ainsi qu'aux rapports de ces dernières avec leurs parties intéressées internes et externes ; ce processus est méthodique, indépendant et documenté, il permet d'obtenir des preuves d'audit et de les évaluer de manière objective pour déterminer dans quelle mesure les critères d'audit sont satisfaits ». L'une de ses missions est d'évaluer les risques sociaux, d'apprécier la vulnérabilité de l'organisation et de mettre le point sur les conséquences dégénérées.

L'audit social est donc une partie d'audit interne, axée sur les RH. Il sert à évaluer leurs activités et leurs performances, dans l'objectif de mener des actions correctives, et ce, à travers une démarche objective pour garantir l'efficacité

Guerrerou (2008) propose la définition suivante : l'audit social est une démarche d'observation, d'analyse et d'évaluation, qui permet d'identifier les points forts et les risques de la gestion des ressources humaines.

\section{Missions de l'audit social}

L'institut international de l'audit social (IAS) a publié en 2006 son référentiel normatif qui définit les quatre principales missions auxquelles répond l'audit social :

1). La vérification de la conformité d'un système social ou de management aux règles et normes nationales ou internationales.

2). L'évolution de l'efficacité et de l'efficience de ce système, et des risques encourus.

3) L'appréciation de la cohérence des politiques sociales et des moyens mis en œuvre.

4) La vérification de la faisabilité socio-économique d'un projet ou d'un programme.

L'audit social permet à la gouvernance de l'entreprise de s'assurer de la conformité de ladite entreprise aux normes en vigueur, mais également du respect de sa stratégie pour tous les aspects qui concernent le champ social. Ainsi, par exemple, les éléments suivants peuvent entrer dans le champ d'un audit social :

- des investigations sur la politique et les pratiques de l'entreprise en matière de diversité ; 
- des éclairages sur les fourchettes de rémunérations des différentes catégories de personnel ;

- un diagnostic sur le capital humain de l'entreprise, ou un rapport sur son évolution souhaitée.

L'I.A.S. définit l'audit social comme « une forme d'observation qui tend à vérifier qu'une organisation a effectivement réalisé ce qu'elle dit avoir fait, qu'elle utilise au mieux ses moyens, qu'elle conserve son autonomie et son patrimoine, qu'elle est capable de réaliser ce qu'elle dit vouloir faire... et [qu'elle] sait évaluer les risques qu'elle court.

\section{Le champ d'application de l'audit social}

Pour les champs d'application de l'audit social, il est possible d'en relever quatre :

- l'audit du social évaluant la conformité des pratiques internes à un référentiel normatif externe (Droit du travail, Conventions Collectives, Accords d'entreprise) et au delà des frontières de l'entreprise (pour les soustraitants et fournisseurs), à des normes liées à la responsabilité sociale de l'entreprise, souvent référencées sur les principes de l'Organisation Internationale du Travail ;

- l'audit de conformité et de pertinence d'une situation, en référence à des objectifs, règles, et procédures internes à l'entreprise. L'entreprise souhaite évaluer la réalité des pratiques par rapport à ce qui est prévu. Elle désire aussi faire évoluer ces pratiques en évaluant dans un premier temps la pertinence des objectifs et processus existants. Les domaines d'application sont nombreux: recrutement, formation, appréciation, gestion des compétences, rémunérations, sécurité du travail, etc.... Ce type d'audit peut intégrer des approches telle que benchmarking afin de permettre des comparaisons externes ;

- l'audit préalable à une situation de changement (fusion-acquisition, réorganisation, faisabilité sociale d'un investissement, (re)négociation d'un accord collectif, etc...) ;

- l'audit permettant la compréhension d'une situation sociale particulière (conflit social, climat social dégradé, démissions, ...), dans le but d'en tirer des enseignements correctifs pour l'avenir et/ou de prévenir la répétition de situations conflictuelles.

\section{Les documents utilisés lors l'audit social}

Les auditeurs sociaux lors de l'audit social peuvent recourir à l'utilisation des documents suivants :

- les documents des représentants du personnel : comme les procèsverbaux, les comptes rendus et rapports produits ou possédés par les 
représentants du personnel dans le but de connaître l'ambiance sociale de l'entreprise ;

- le bilan social : permet à l'auditeur de disposer des informations concernant :

- $\quad$ la structure de la population à savoir la répartition par âge, par sexe, le degré de qualification et d'encadrement ;

- la qualité de vie au travail en termes de conditions physiques de travail, de la durée et de l'aménagement du temps du travail ainsi que les conditions d'hygiène et de sécurité ;

- les comportements du personnel mesurés notamment par les taux d'absentéisme, turnover et de départs ;

- $\quad$ l'examen des politiques sociales de l'entreprise ;

- la politique de formation.

- les documents sociaux (conventions collectives, règlement intérieur) : l'élaboration de ces documents est imposée par les articles 104 et $138 \mathrm{du}$ code de travail en vigueur. L'auditeur doit ici s'assurer de la conformité de ces documents aux textes en vigueur ;

- l'enquête opinion : est une recherche des aspirations du personnel par sondage et utilisée pour mesurer le degré d'adhésion du personnel aux projets et aux objectifs de l'entreprise.

\section{Les outils de l'audit social}

\section{Les outils utilisés en amont de la mission}

Parmi les outils d'audit préalables au travail terrain, on cite les questionnaires de prise de connaissance (QPC), les questionnaires de contrôle interne (QCI), le tableau d'attribution des responsabilités (TAR) et les plans d'échantillonnage.

- Le QPC est destiné préparer l'arrivée sur le site de l'auditeur social et la première réunion (la réunion de lancement). Il consiste à demander des informations et des documents dont l'auditeur prendra connaissance in situ.

Le QPC (Combemale et Igalens, 2005) peut se présenter sous une forme simple de type page divisée en trois ou quatre colonnes : la première est consacrée à un numéro, la seconde à une question formulée par l'auditeur, la troisième à la réponse précise rédigée par l'audité et la quatrième aux remarques de l'un ou l'autre.

Les informations à récolter peuvent être regroupées en différents thèmes :

- $\quad$ contexte structurel de l'entité auditée ;

- $\quad$ structure et organisation internes de l'entité auditée ;

- $\quad$ organigramme et relations de pouvoirs notamment les délégations ;

- environnement informatique ; 
- $\quad$ contexte réglementaire ;

- $\quad$ processus et procédures ;

- $\quad$ système d'information : communication interne et externe ;

- $\quad$ problèmes passés ou en cours ;

- $\quad$ réformes en cours ou prévues.

- Le QCI a pour objectif d'orienter les travaux de l'auditeur vers les objectifs de la mission et notamment les opérations les moins bien maîtrisées. Il est composé de questions fermées, posées dans un ordre logique (souvent chronologique), formulées de telle sorte que la réponse oui corresponde à un contrôle interne satisfaisant et la réponse non à un contrôle interne insatisfaisant (absent, défaillant, inapproprié au risque), et enfin assorties de possibilités de commentaires de la part de l'audité.

Le QCI contient cinq questions fondamentales qui permettent de regrouper l'ensemble des interrogations concernant les points de contrôle :

- Qui ? Questions relatives à l'opérateur qu'il faut identifier avec précision pour déterminer quels sont ses pouvoirs.

- Quoi ? Questions relatives à l'objet de l'opération, quelle est la nature de la tâche, du produit fabriqué, du contrôle.

- $\quad$ Où ? Concerne les endroits où se déroule l'opération.

- $\quad$ Quand ? Questions relatives au temps : début, fin, durée,...

- Comment? Questions relatives à la description au mode opératoire, comment se réalise la tâche.

- Les TAR (Combemale et Igalens, 2005) se présentent souvent sous forme de tableaux à double entrée, ligne et colonne. En colonne, figurent les activités de base d'un processus et en ligne, les différentes personnes (ou bien les différents postes) qui réalisent ces activités. Au croisement ligne/colonne, on utilise un code qui permet de préciser la nature de la responsabilité de la personne par rapport à l'activité. Ainsi un code précisera si la personne : prévoit ou planifie (P), décide (D), exécute $(\mathrm{E})$, contrôle $(\mathrm{C})$.

- Les plans d'échantillonnage (Igalens et Peretti, 2008) sont de nature différente en fonction des objectifs de l'audit. L'auditeur a recours aux techniques d'échantillonnage statistiques lorsqu'il n'aura pas le temps ou les moyens pour mener à bien une analyse exhaustive de l'information dont il dispose. La première étape de sa démarche consiste à choisir une méthode d'échantillonnage en fonction du problème étudié et de l'information dont il dispose. Après avoir constitué son échantillon, il analyse et évalue les résultats obtenus sur celui-ci.

\section{Les outils utilisés pendant la mission}

Parmi les outils les plus importants utilisés pendant la mission d'audit, figurent l'entretien et l'observation et les flow chart. 
- L'entretien et l'observation: l'entretien dans le cadre de l'audit social peut être différent selon les étapes et les objectifs de l'audit. On peut distinguer des entretiens individuels et des entretiens de groupe, on peut également opposer des entretiens ayant pour objectif d'obtenir des informations précises sur des données, des processus, des habitudes de travail et des entretiens orientés vers du vécu, des perceptions et de opinions des personnes interrogées.

L'observation est une évidence pour l'auditeur social, qu'il s'agisse des conditions de travail, du climat social, du respect des règles du droit de travail. Sur ces points l'auditeur doit faire preuve de curiosité

- Le flow chart ou le diagramme de circulation des documents (Combemale et Igalens, 2005) est un tableau qui permet de visualiser les flux de traitement de l'information et de documents, il permet de voir d'où ils proviennent, qui les saisit, les traite, les vérifie, les stocke. Cet outil permet également de faire des vérifications comme par exemple le destinataire des exemplaires et la conformité des informations.

\section{Méthodologie}

La démarche exploratoire adoptée dans cette recherche justifie le recours à une méthodologie qualitative. Cette méthodologie se résume en des entretiens semi-directifs menés auprès du personnel de deux entités la commune urbaine d'Agadir (CUA) et la société Moyen et Grand Atlas (MGA) qui exerce ses activités dans le domaine de la construction et bâtiments d'entreprises à travers un questionnaire composé de 9 rubriques.

\section{Résultats et discussions}

Les résultats de l'enquête terrain ont été comme suit :

- Le recrutement

\begin{tabular}{|c|c|c|c|c|}
\hline Organisation & Questions & Choix & $\begin{array}{c}\text { Tot } \\
\text { al }\end{array}$ & $\begin{array}{c}\text { Pourcen } \\
\text { tage }\end{array}$ \\
\hline \multirow{6}{*}{$\begin{array}{c}\text { Commune Urbaine } \\
\text { d'Agadir (CUA) }\end{array}$} & \multirow{3}{*}{$\begin{array}{l}\text { Le processus de recrutement est-il } \\
\text { satisfaisant? }\end{array}$} & Non & 6 & $30 \%$ \\
\hline & & $\begin{array}{c}\text { Plutôt } \\
\text { satisfaisant }\end{array}$ & 3 & $15 \%$ \\
\hline & & $\begin{array}{c}\text { Très } \\
\text { satisfaisant }\end{array}$ & 11 & $55 \%$ \\
\hline & \multirow{3}{*}{$\begin{array}{c}\text { Ce processus permet-il d'attirer du } \\
\text { personnel qualifié ? }\end{array}$} & Non & 3 & $15 \%$ \\
\hline & & $\begin{array}{c}\text { Plutôt } \\
\text { satisfaisant }\end{array}$ & 14 & $70 \%$ \\
\hline & & $\begin{array}{c}\text { Très } \\
\text { satisfaisant }\end{array}$ & 3 & $15 \%$ \\
\hline \multirow{3}{*}{$\begin{array}{l}\text { Moyen et Grand } \\
\text { ATLAS (MGA) }\end{array}$} & \multirow{3}{*}{$\begin{array}{l}\text { Le processus de recrutement est-il } \\
\text { satisfaisant? }\end{array}$} & Non & 4 & $20 \%$ \\
\hline & & $\begin{array}{c}\text { Plutôt } \\
\text { satisfaisant }\end{array}$ & 11 & $55 \%$ \\
\hline & & Très & 5 & $25 \%$ \\
\hline
\end{tabular}




\begin{tabular}{|l|c|c|c|c|} 
& & satisfaisant & & \\
\cline { 2 - 5 } & \multirow{4}{*}{$\begin{array}{c}\text { Ce processus permet-il d'attirer du } \\
\text { personnel qualifié ? }\end{array}$} & $\begin{array}{c}\text { Plutôt } \\
\text { satisfaisant }\end{array}$ & 10 & $20 \%$ \\
\cline { 3 - 5 } & $\begin{array}{c}\text { Très } \\
\text { satisfaisant }\end{array}$ & 6 & $30 \%$ \\
\hline
\end{tabular}

Selon le personnel de la CUA, le recrutement se fait par concours et d'après le tableau ci-dessus on observe que la majorité des recrutés sont satisfaits de ce processus appliqué au sein de cette commune, et qui lui permet d'acquérir des ressources humaines qualifiées. Egalement pour les salariés du MGA qui sont recrutés selon des entretiens, et qui sont plutôt satisfaits de ce processus.

- L'intégration

\begin{tabular}{|c|c|c|c|c|}
\hline Organisation & Questions & Choix & $\begin{array}{l}\mathrm{T} \\
\text { ot } \\
\mathrm{al}\end{array}$ & $\begin{array}{l}\text { Pourc } \\
\text { entag } \\
\text { e }\end{array}$ \\
\hline \multirow{7}{*}{$\begin{array}{l}\text { Commune } \\
\text { Urbaine d'Agadir } \\
\text { (CUA) }\end{array}$} & \multirow{3}{*}{$\begin{array}{l}\text { L'organisation applique-t-elle un processus } \\
\text { d'accueil et d'intégration des nouveaux } \\
\text { salariés? }\end{array}$} & Non & 14 & $70 \%$ \\
\hline & & $\begin{array}{c}\text { Plutôt } \\
\text { satisfaisant }\end{array}$ & 6 & $30 \%$ \\
\hline & & Très satisfaisant & 0 & $0 \%$ \\
\hline & \multirow{4}{*}{$\begin{array}{l}\text { L'accueil officiel des nouveaux salariés se } \\
\text { fait à travers : }\end{array}$} & $\begin{array}{l}\text { Rencontre avec } \\
\text { les responsables }\end{array}$ & 9 & $45 \%$ \\
\hline & & Visites guidées & 2 & $10 \%$ \\
\hline & & $\begin{array}{l}\text { Rencontre des } \\
\text { collaborateurs }\end{array}$ & 4 & $20 \%$ \\
\hline & & Autres & 5 & $25 \%$ \\
\hline \multirow{7}{*}{$\begin{array}{l}\text { Moyen et Grand } \\
\text { Atlas (MGA) }\end{array}$} & \multirow{3}{*}{$\begin{array}{l}\text { L'organisation applique-t-elle un processus } \\
\text { d'accueil et d'intégration des nouveaux } \\
\text { salariés? }\end{array}$} & Non & 5 & $25 \%$ \\
\hline & & $\begin{array}{c}\text { Plutôt } \\
\text { satisfaisant }\end{array}$ & 7 & $35 \%$ \\
\hline & & Très satisfaisant & 8 & $40 \%$ \\
\hline & \multirow{4}{*}{$\begin{array}{c}\text { L'accueil officiel des nouveaux salariés se fait } \\
\text { à travers : }\end{array}$} & $\begin{array}{l}\text { Rencontre avec } \\
\text { les responsables }\end{array}$ & 13 & $65 \%$ \\
\hline & & Visites guidées & 0 & $0 \%$ \\
\hline & & $\begin{array}{l}\text { Rencontre des } \\
\text { collaborateurs }\end{array}$ & 2 & $10 \%$ \\
\hline & & Autres & 5 & $25 \%$ \\
\hline
\end{tabular}

D'après ce tableau on remarque que les deux organisations suivent la même méthode d'accueil de leurs nouveaux recrus (rencontre avec les responsables). Cette méthode qui est satisfaisante pour les salariés de MGA, cependant elle est inutile pour le personnel de la CUA dans l'absence d'un processus d'intégration des nouveaux salariés.

Les deux organisations appliquent la même méthode d'accueil des nouveaux recrutés dans la plupart des fois (rencontre avec les responsables) en plus qu'elles suivent d'autres méthodes pour d'autres cas.

- La rémunération 


\begin{tabular}{|c|c|c|c|c|}
\hline Organisation & Questions & Choix & $\begin{array}{l}\text { To } \\
\text { tal }\end{array}$ & $\begin{array}{c}\text { Pource } \\
\text { ntage }\end{array}$ \\
\hline \multirow{6}{*}{$\begin{array}{l}\text { Commune Urbaine } \\
\text { d'Agadir (CUA) }\end{array}$} & \multirow{3}{*}{$\begin{array}{l}\text { Votre rémunération est-elle } \\
\text { satisfaisante et bien définie? }\end{array}$} & Non & 6 & $30 \%$ \\
\hline & & $\begin{array}{c}\text { Plutôt } \\
\text { satisfaisant }\end{array}$ & 14 & $70 \%$ \\
\hline & & $\begin{array}{c}\text { Très } \\
\text { satisfaisant } \\
\end{array}$ & 0 & $0 \%$ \\
\hline & \multirow{3}{*}{ Votre salaire est-il satisfaisant? } & Non & 7 & $35 \%$ \\
\hline & & $\begin{array}{c}\text { Plutôt } \\
\text { satisfaisant }\end{array}$ & 12 & $60 \%$ \\
\hline & & $\begin{array}{c}\text { Très } \\
\text { satisfaisant }\end{array}$ & 1 & $5 \%$ \\
\hline \multirow{6}{*}{$\begin{array}{l}\text { Moyen et Grand Atlas } \\
\text { (GMA) }\end{array}$} & \multirow{3}{*}{$\begin{array}{l}\text { Votre rémunération est-elle } \\
\text { satisfaisante et bien définie ? }\end{array}$} & Non & 5 & $25 \%$ \\
\hline & & $\begin{array}{c}\text { Plutôt } \\
\text { satisfaisant }\end{array}$ & 10 & $50 \%$ \\
\hline & & $\begin{array}{c}\text { Très } \\
\text { satisfaisant } \\
\end{array}$ & 5 & $25 \%$ \\
\hline & \multirow{3}{*}{ Votre salaire est-il satisfaisant? } & Non & 3 & $15 \%$ \\
\hline & & $\begin{array}{c}\text { Plutôt } \\
\text { satisfaisant }\end{array}$ & 12 & $60 \%$ \\
\hline & & $\begin{array}{c}\text { Très } \\
\text { satisfaisant } \\
\end{array}$ & 5 & $25 \%$ \\
\hline
\end{tabular}

leur rémunération.

La majorité du personnel des deux organisations sont satisfaits de

\section{- La formation}

\begin{tabular}{|c|c|c|c|c|}
\hline Organisation & Questions & Choix & $\begin{array}{l}\text { To } \\
\text { tal }\end{array}$ & $\begin{array}{c}\text { Pource } \\
\text { ntage }\end{array}$ \\
\hline \multirow{8}{*}{$\begin{array}{l}\text { Commune Urbaine } \\
\text { d'Agadir (CUA) }\end{array}$} & \multirow{3}{*}{$\begin{array}{l}\text { Bénéficiez-vous d'une } \\
\text { formation professionnelle ? }\end{array}$} & non & 14 & $70 \%$ \\
\hline & & Plutôt satisfaisant & 5 & $25 \%$ \\
\hline & & Très satisfaisant & 1 & $5 \%$ \\
\hline & \multirow{5}{*}{$\begin{array}{l}\text { Vos raisons de suivre une } \\
\text { formation : }\end{array}$} & La promotion & 3 & $15 \%$ \\
\hline & & $\begin{array}{c}\text { L'acquisition de } \\
\text { nouvelles compétences }\end{array}$ & 3 & $15 \%$ \\
\hline & & L'évolution du métier & 12 & $60 \%$ \\
\hline & & Les raisons matérielles & 0 & $0 \%$ \\
\hline & & Autres & 2 & $10 \%$ \\
\hline \multirow{8}{*}{$\begin{array}{l}\text { Moyen et Grand Atlas } \\
\text { (GMA) }\end{array}$} & \multirow{3}{*}{$\begin{array}{l}\text { Bénéficiez-vous d'une } \\
\text { formation professionnelle? }\end{array}$} & non & 10 & $50 \%$ \\
\hline & & Plutôt satisfaisant & 8 & $40 \%$ \\
\hline & & Très satisfaisant & 2 & $10 \%$ \\
\hline & \multirow{5}{*}{$\begin{array}{l}\text { Vos raisons de suivre une } \\
\text { formation? }\end{array}$} & La promotion & 2 & $10 \%$ \\
\hline & & $\begin{array}{c}\text { L'acquisition de } \\
\text { nouvelles compétences }\end{array}$ & 0 & $0 \%$ \\
\hline & & L'évolution du métier & 8 & $40 \%$ \\
\hline & & Les raisons matérielles & 0 & $0 \%$ \\
\hline & & Autres & 10 & $50 \%$ \\
\hline
\end{tabular}


D'après le tableau ci-dessus on observe que l'évolution de métier est la principale raison qui pousse plus à vouloir suivre une formation ce qui montre que le personnel s'intéresse plus à la carrière professionnelle.

On remarque aussi au sein de la commune urbaine que la majorité des employés $(70 \%)$ ne bénéficient pas d'une formation, cependant la société Moyen et Grand Atlas organise des formations plutôt satisfaisantes à une tranche importante $(40 \%)$ de ses employés.

Concernant la formation, la CUA ne suit pas un rythme de formation régulier, on peut dire qu'elle néglige ce concept.

- La circulation de l'information

\begin{tabular}{|c|c|c|c|c|}
\hline Organisation & Questions & Choix & $\begin{array}{l}\text { To } \\
\text { tal }\end{array}$ & $\begin{array}{l}\text { Pource } \\
\text { ntages }\end{array}$ \\
\hline \multirow{7}{*}{$\begin{array}{l}\text { Commune Urbaine } \\
\text { d'Agadir (CUA) }\end{array}$} & \multirow{4}{*}{$\begin{array}{l}\text { Les modes de circulation de } \\
\text { l'information se font par: }\end{array}$} & Par avis & 10 & $50 \%$ \\
\hline & & $\begin{array}{c}\text { Par } \\
\text { internet }\end{array}$ & 3 & $15 \%$ \\
\hline & & $\begin{array}{l}\text { Par } \\
\text { réunion }\end{array}$ & 5 & $25 \%$ \\
\hline & & Autres & 2 & $10 \%$ \\
\hline & \multirow{3}{*}{$\begin{array}{l}\text { L'avis et les propositions du personnel } \\
\text { sont-ils pris en considération? }\end{array}$} & Non & 10 & $50 \%$ \\
\hline & & $\begin{array}{c}\text { Plutôt } \\
\text { satisfaisa } \\
\text { nt }\end{array}$ & 10 & $50 \%$ \\
\hline & & $\begin{array}{c}\text { Très } \\
\text { satisfaisa } \\
\text { nt } \\
\end{array}$ & 0 & $0 \%$ \\
\hline \multirow{7}{*}{$\begin{array}{l}\text { Moyen et Grand } \\
\text { Atlas (GMA) }\end{array}$} & \multirow{4}{*}{$\begin{array}{l}\text { Les modes de circulation de } \\
\text { l'information se font par: }\end{array}$} & Par avis & 5 & $25 \%$ \\
\hline & & $\begin{array}{c}\text { Par } \\
\text { internet }\end{array}$ & 1 & $5 \%$ \\
\hline & & $\begin{array}{c}\text { Par } \\
\text { réunion }\end{array}$ & 12 & $60 \%$ \\
\hline & & Autres & 2 & $10 \%$ \\
\hline & \multirow{3}{*}{$\begin{array}{l}\text { L'avis et les propositions du personnel } \\
\text { sont-ils pris en considération? }\end{array}$} & Non & 3 & $15 \%$ \\
\hline & & $\begin{array}{c}\text { Plutôt } \\
\text { satisfaisa } \\
\text { nt }\end{array}$ & 6 & $30 \%$ \\
\hline & & $\begin{array}{c}\text { Très } \\
\text { satisfaisa } \\
\text { nt }\end{array}$ & 11 & $55 \%$ \\
\hline
\end{tabular}

L'information circule au sein de CUA par avis ce qui rend $50 \%$ de personnel satisfait mais non pas la totalité. Mais pour MGA, le personnel est informé selon des réunions après par avis, ce qui montre l'existence de la communication interne entre les employés en général au sein cette société.

- Le climat social 


\begin{tabular}{|c|c|c|c|c|}
\hline Organisation & Questions & Choix & $\begin{array}{l}\text { To } \\
\text { tal }\end{array}$ & $\begin{array}{l}\text { Pource } \\
\text { ntages }\end{array}$ \\
\hline \multirow{9}{*}{$\begin{array}{l}\text { Commune urbaine } \\
\text { d'Agadir (CUA) }\end{array}$} & \multirow{3}{*}{ Le taux des conflits est-il? } & Faible & 15 & $75 \%$ \\
\hline & & Assez fort & 3 & $15 \%$ \\
\hline & & Très fort & 2 & $10 \%$ \\
\hline & \multirow{2}{*}{$\begin{array}{l}\text { Existe-il des représentants du } \\
\text { personnel dans l'organisation? }\end{array}$} & Oui & 20 & $100 \%$ \\
\hline & & Non & 0 & $0 \%$ \\
\hline & \multirow{4}{*}{$\begin{array}{c}\text { Les conflits sont réglés du } \\
\text { quelle manière? }\end{array}$} & $\begin{array}{l}\text { Recours au } \\
\text { représentant du } \\
\text { personnel }\end{array}$ & 7 & $35 \%$ \\
\hline & & $\begin{array}{c}\text { Par plainte au } \\
\text { supérieur }\end{array}$ & 10 & $50 \%$ \\
\hline & & Par ignorance & 1 & $5 \%$ \\
\hline & & Autres & 2 & $10 \%$ \\
\hline \multirow{9}{*}{$\begin{array}{l}\text { Moyen et Grand } \\
\text { Atlas (GMA) }\end{array}$} & \multirow{3}{*}{ Le taux des conflits est-il? } & Faible & 17 & $85 \%$ \\
\hline & & Assez fort & 2 & $10 \%$ \\
\hline & & Très fort & 1 & $5 \%$ \\
\hline & \multirow{2}{*}{$\begin{array}{l}\text { Existe-il des représentants du } \\
\text { personnel dans l'organisation? }\end{array}$} & Oui & 2 & $10 \%$ \\
\hline & & Non & 18 & $90 \%$ \\
\hline & \multirow{4}{*}{$\begin{array}{c}\text { Les conflits sont réglés du } \\
\text { quelle manière? }\end{array}$} & $\begin{array}{l}\text { Recours au } \\
\text { représentant du } \\
\text { personnel }\end{array}$ & 1 & $5 \%$ \\
\hline & & $\begin{array}{l}\text { Par plainte au } \\
\text { supérieur }\end{array}$ & 13 & $65 \%$ \\
\hline & & Par ignorance & 0 & $0 \%$ \\
\hline & & Autres & 6 & $30 \%$ \\
\hline
\end{tabular}

D'après les résultats de l'enquête on remarque que la majorité des employés des deux organisations règlent leurs problèmes par plainte au supérieur, et seulement $35 \%$ des employés de la CUA qui recourent aux services des représentants du personnel. Et on constate que la société MGA n'a pas des représentants des employés.

A ce niveau les employés de la CUA travaillent en groupe, dans des fonctions spécifiques (service d'achat, service social, service de division des affaires sociales...) avec un chef bien déterminé. Aussi les salariés de MGA travaillent en groupe, géré par un chef et s'il y'a un conflit c'est au chef de service ou bien le gérant de réagir en utilisant des outils de sanction. 


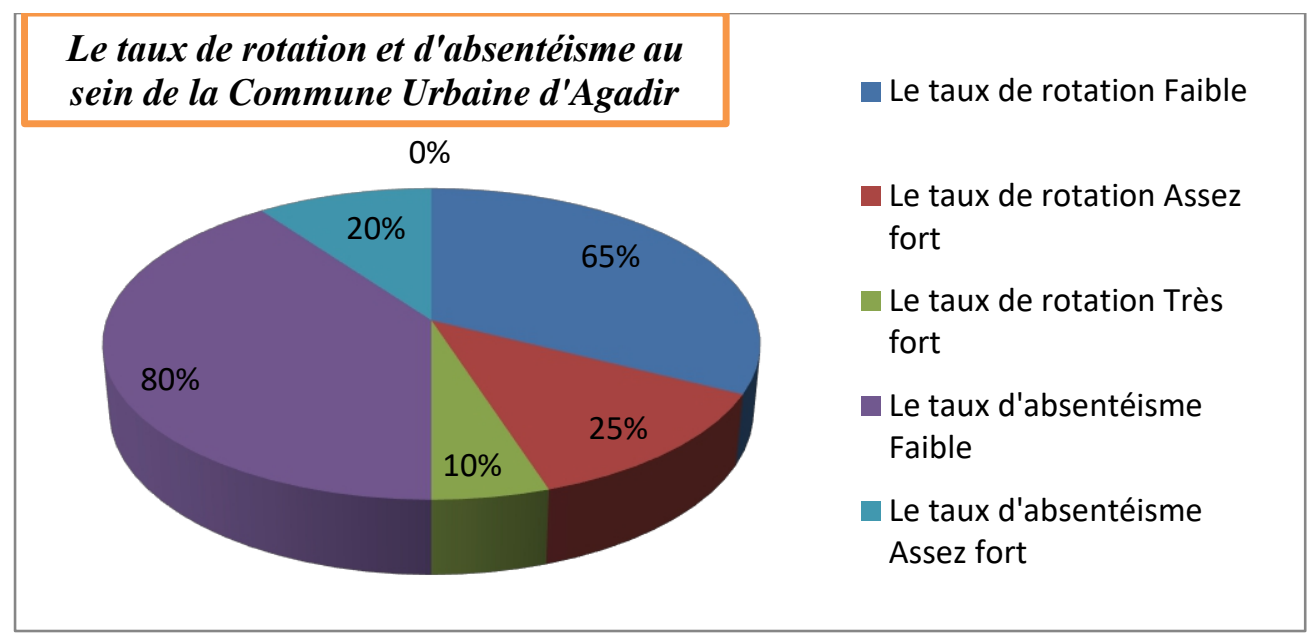

Selon le graphique ci-dessus, on constate que la plupart des employés de la CUA sont disciplinés sachant que $80 \%$ des employés considèrent que le taux d'absentéisme est faible, concernant le taux de rotation, $65 \%$ du personnel le voit aussi faible.

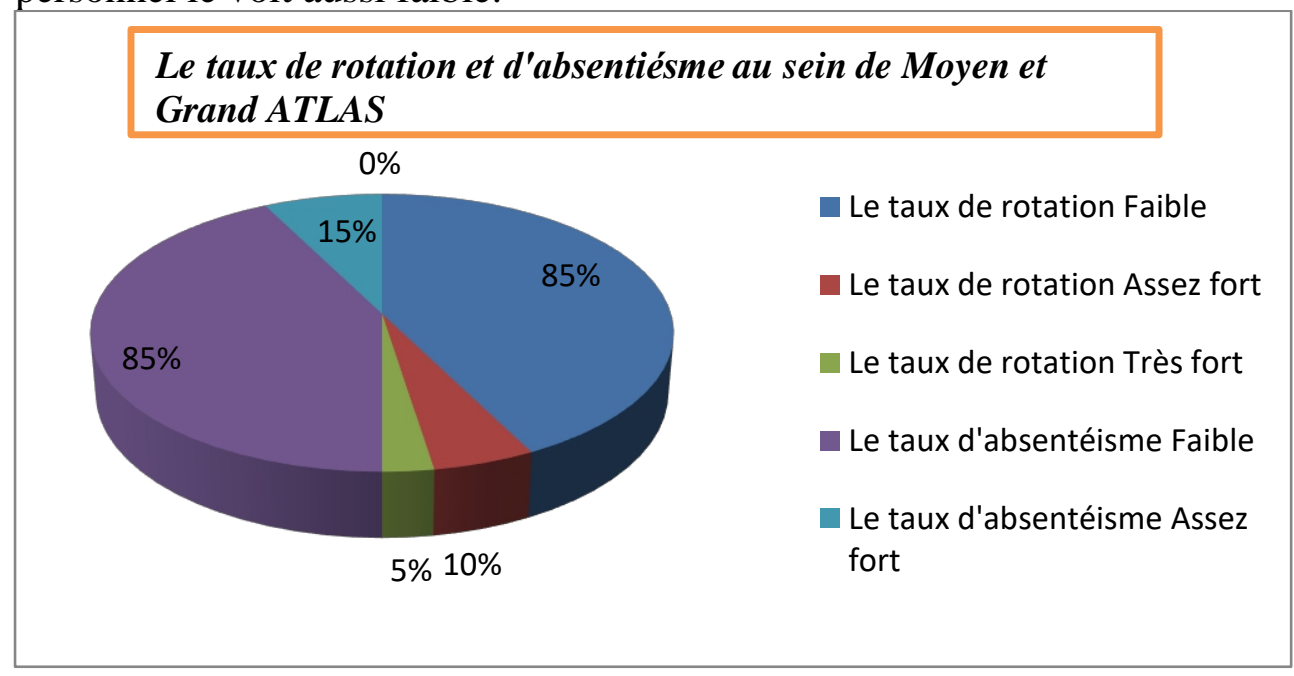

Pour la société MGA, $85 \%$ de ses employés pensent que le taux de rotation et d'absentéisme sont faibles et $15 \%$ considèrent le taux d'absentéisme comme assez fort.

Pour les deux organisations, en cas de non justification de l'absence on demande des explications aux absents et on suit la procédure de gestion des absences.

- La gestion des carrières 


\begin{tabular}{|c|c|c|c|c|}
\hline Organisation & Questions & Choix & $\begin{array}{l}\text { To } \\
\text { tal }\end{array}$ & $\begin{array}{l}\text { Pourc } \\
\text { entage }\end{array}$ \\
\hline \multirow{6}{*}{$\begin{array}{l}\text { commune urbaine } \\
\text { d'Agadir (CUA) }\end{array}$} & \multirow{3}{*}{ Bénéficiez-vous des promotions? } & non & 12 & $60 \%$ \\
\hline & & $\begin{array}{c}\text { Plutôt } \\
\text { satisfaisa } \\
\text { nt }\end{array}$ & 6 & $30 \%$ \\
\hline & & $\begin{array}{c}\text { Très } \\
\text { satisfaisa } \\
\text { nt }\end{array}$ & 2 & $10 \%$ \\
\hline & \multirow{3}{*}{$\begin{array}{l}\text { Votre carrière se développe-t-elle au cours } \\
\text { de votre parcours professionnel ? }\end{array}$} & Non & 3 & $15 \%$ \\
\hline & & $\begin{array}{c}\text { Plutôt } \\
\text { satisfaisa } \\
\text { nt }\end{array}$ & 16 & $80 \%$ \\
\hline & & $\begin{array}{c}\text { Très } \\
\text { satisfaisa } \\
\text { nt }\end{array}$ & 1 & $5 \%$ \\
\hline \multirow{6}{*}{$\begin{array}{l}\text { Moyen et Grand } \\
\text { Atlas (GMA) }\end{array}$} & \multirow{3}{*}{ Bénéficiez-vous des promotions? } & Non & 12 & $60 \%$ \\
\hline & & $\begin{array}{c}\text { Plutôt } \\
\text { satisfaisa } \\
\text { nt }\end{array}$ & 8 & $40 \%$ \\
\hline & & $\begin{array}{c}\text { Très } \\
\text { satisfaisa } \\
\text { nt }\end{array}$ & 0 & $0 \%$ \\
\hline & \multirow{3}{*}{$\begin{array}{l}\text { Votre carrière se développe-t-elle au cours } \\
\text { de votre parcours professionnel ? }\end{array}$} & Non & 6 & $30 \%$ \\
\hline & & $\begin{array}{c}\text { Plutôt } \\
\text { satisfaisa } \\
\text { nt }\end{array}$ & 11 & $55 \%$ \\
\hline & & $\begin{array}{c}\text { Très } \\
\text { satisfaisa } \\
\text { nt }\end{array}$ & 3 & $15 \%$ \\
\hline
\end{tabular}

Concernant la promotion au sein des deux organisations on retient que $40 \%$ des employés ont profité d'une promotion par contre $60 \%$ n'ont pas pu en bénéficier. Et pourtant la carrière de la majorité des employés s'améliore tout au long leurs parcours professionnel.

- La gestion des données du personnel

La CUA ne dispose pas d'un système d'informations sociales fiable et pertinent ce qui ne lui permet pas de bien gérer son personnel. Cependant que la société MGA possède d'un système d'informations sociales mais qui n'est pas actualisé régulièrement.

Les informations sociales doivent être fiables et bien déterminées pour faciliter la tâche de gestion des ressources humaines au sein des deux organisations. Les deux organisations doivent améliorer le système social.

- La valorisation du personnel

Les deux organisations ne disposent pas d'un budget important pour la valorisation de leurs ressources humaines, aussi elles n'appliquent pas un système déterminé de rémunération et d'avantages. 
Pour la motivation, les deux organisations doivent procéder à la mise en place d'un système de motivation pour encourager plus leurs employés.

D'une façon générale, l'enquête a permis de constater que l'introduction de l'audit social dans les organisations est nécessaire puisque ce dernier a un effet bénéfique sur la gestion des ressources humaines dans la mesure où il veille à garder un climat social positif.

L'audit social est un outil destiné aux hautes instances des organisations puisqu'il permet de : humaines ;

- relever d'éventuels dysfonctionnements par rapport aux lois et législations en vigueur en matière de gestion des ressources humaines ;

- $\quad$ fournir des analyses objectives sur la gestion des ressources humaines et des recommandations pertinentes pour améliorer les conditions du travail et les approches de management du capital humain.

L'intégration de l'audit social dans les organisations pour piloter les ressources humaines s'avère nécessaire pour les raisons suivantes :

- $\quad$ améliorer la qualité du pilotage social ;

- $\quad$ assurer une GRH pertinente ;

- $\quad$ rentabiliser les emplois ;

- $\quad$ renforcer le partenariat avec les différents organes sociaux ;

- $\quad$ mettre en place une stratégie sociale permettant la consolidation de la paix sociale.

\section{Conclusion}

L'introduction de l'audit social dans la culture de certaines organisations marocaines reste limitée malgré l'importance qu'il joue dans la gestion des ressources humaines. Cette limite est de nature culturelle, financière et technique.

Avec l'ouverture du Maroc sur l'étranger, l'implantation des sociétés étrangères et l'amélioration du niveau d'instruction des employés, le Maroc est invité à consolider la pratique de l'audit social par les organisations publiques et privées pour améliorer la qualité du pilotage social et renforcer les relations de partenariat avec les instances opérant dans le domaine social.

\section{References:}

1. Barney, J. (1991). Firm Resources and Sustained Competitive Advantage, Journal of Management, (17), p.99-120.

2. Candau P. (1985). Audit social, Edition Vuibert, Paris.

3. Combemale, M. et Igalens, J. (2005). L'audit social. Que sais-je ? PUF. 
4. Haegel, A. (2010). Toute la fonction ressource Humaine. Paris: Dunod.

5. Guerrero S. (2004). Les outils des ressources humaines, Dunod, Paris.

6. Guerrero S. (2008). Les outils de l'audit social : optimiser la gestion des ressources humaines, Dunod, Paris.

7. Ireland, R.D. et Hitt, M.A. (2002). Alliance Management as a Source of Competitive Advantage, Journal of Management, 28 (3): 413-446.

8. Igalens, J. (2000). Audit des ressources humaines. Editions Liaisons : France.

9. Igalens, J. et Peretti, J-M. (2008). Audit social : Meilleures pratiques, méthodes, outils. Ed Eyrolles : Paris.

10. Penrose, E. (1959). The theory of the growth of the firm, Oxford University Press. 4th Ed.

11. Mahoney, J. (1995). The management of Resources and the Resource of Management, Journal of Business Research, (33): 91-101.

12. Vatier R. (1980). L'audit social, un instrument utile au pilotage des entreprises et des organisations, Enseignement et Gestion, $\mathrm{n}^{\circ} 16$. 